\title{
Evaluation of hypertension treatment in acute ischemic stroke
}

\author{
Lara Haidar $^{\text {a, Hiba AlHarfany }}{ }^{\text {a }}$, Sarah G. Cherri ${ }^{\text {a, *, Diana Malaeb }}{ }^{\text {a,f }}$, Nada Dia ${ }^{\text {, }}$ \\ Pascale Salameh ${ }^{\text {b,c,d }}$, Hassan Hosseini ${ }^{\text {e, }}$ \\ a School of Pharmacy, Lebanese International University, Beirut, Lebanon \\ ${ }^{\mathrm{b}}$ INSPECT-LB: Institut National de Santé Publique, Epidémiologie Clinique et Toxicologie, Lebanon \\ ${ }^{\mathrm{c}}$ Faculty of Pharmacy, Lebanese University, Hadat, Lebanon \\ ${ }^{\mathrm{d}}$ University of Nicosia Medical School, Nicosia, Cyprus \\ ${ }^{\text {e }}$ Stroke Unit, Service de Neurologie, CHU Henri Mondor, France \\ ${ }^{\mathrm{f}}$ Université Paris-Est Créteil, Faculté de Santé, France
}

\section{A R T I C L E I N F O}

\section{Keywords:}

Stroke

Blood pressure

Treatment

Guidelines

\begin{abstract}
A B S T R A C T
Background: Treating hypertension (HTN) in acute ischemic stroke (AIS) is controversial, since both extremely high and low blood pressures (BP) are associated with poor outcomes.

Objective: This study was designed to describe and assess the frequency and the factors affecting BP overtreatment in AIS.

Methods and material: This was a retrospective observational study conducted at Lebanese hospitals. BP readings at baseline and after treatment with antihypertensive drugs were recorded. Overtreatment was defined as baseline BP below 220/120 $\mathrm{mmHg}$ and an antihypertensive drug was prescribed.

Results: A total of 141 patients were enrolled with a mean age of 76.89 years and $43.3 \%$ were males. Overtreatment rate was $62.6 \%$, and was significantly more common in patients with a previous history of HTN and hyperlipidemia. In addition, overtreatment was significantly more common in patients treated with antihypertensive drugs, had high modified Rankin Scale score $(>2)$ at baseline, and had high systolic BP at baseline ( $\mathrm{p}<$ 0.05). Results of multivariate analysis revealed that the highest risk of overtreatment was significantly shown in patients who had HTN (OR $=4.589, \mathrm{p}=0.017$ ), and who had high systolic BP at baseline $(\mathrm{OR}=1.061, \mathrm{p}=$ 0.001).

Conclusion: BP management in AIS was generally not consistent with the guidelines. This supports the need for further research to investigate the effects of BP overtreatment on patient outcomes.
\end{abstract}

\section{Introduction}

Stroke is a major leading cause of mortality and principal factor responsible for long-term severe disability where the majority is of ischemic origin. ${ }^{1,2}$ Hypertension (HTN) is the most prevalent risk factor for primary stroke development through accelerating the arteriosclerotic process, and aggravating the risk of embolic formation from large extracranial vessels. ${ }^{1}$ HTN is a common early finding in acute ischemic stroke (AIS) affecting around $80 \%$ of the patients. ${ }^{3}$ The activation of the neuro-endocrine system in response to exposed stress, autonomic dysfunction, previous undiagnosed or uncontrolled hypertension are thought to attribute to the acute increase in blood pressure (BP). ${ }^{4,5}$ Treating HTN in the acute setting is controversial, since both high and low BPs are associated with poor outcomes. The rationale for lowering $\mathrm{BP}$ is to reduce cerebral edema and limit hemorrhagic transformation. However, rapid lowering of BP worsens cerebral hypoperfusion, exacerbates stroke symptoms, expands ischemic core, increases size of infarction, and isn't associated with greater therapeutic benefit. ${ }^{6,7}$ Moreover, the treatment of HTN within the first $72 \mathrm{~h}$ after hospital presentation is not considered an effective strategy to minimize mortality. ${ }^{8}$ In addition, there are several published studies that support the fact that increased BP readings are correlated with higher mortality and poor functional outcomes. ${ }^{9-11}$ American Heart Association and American Stroke Association (AHA/ASA) guidelines for the treatment of BP in AIS, recommended that the initial treatment of hypertension should be delayed in the early post-stroke period except if the BP levels $>200 / 110$

\footnotetext{
* Corresponding author.

E-mail addresses: 31230570@students.liu.edu.lb (L. Haidar), alharfanyhiba@gmail.com (H. AlHarfany), sarashere940@gmail.com (S.G. Cherri), diana.malaeb@ liu.edu.lb (D. Malaeb), nadadia29@gmail.com (N. Dia), pascalesalameh1@hotmail.com (P. Salameh), hassan.hosseini@aphp.fr (H. Hosseini).
} 
mmHg associated with end organ damage, and if the patient is candidate for thrombolysis with BP readings above $185 / 110 \mathrm{mmHg} .{ }^{8,12,13}$ In the acute phase of stroke, there is lack of evidence to suggest specific antihypertensive agent over the others and the guidelines recommend that potent lowering of BP within $24 \mathrm{~h}$ post stroke should be evaded. However, it's preferable to use medications that are reversible, titratible, and have a low propensity to cause cerebral edema. The AHA/ASA guidelines recommend the use of intravenous Nicardipine, Labetalol, or Clevidipine. ${ }^{8}$ However, the guidelines recommendation is based on limited evidence and in the absence of clear guidelines, prescription patterns might vary and the exact target levels of BP remains unspecified. ${ }^{14}$ In Lebanon, there are various studies that are conducted and aimed to evaluate the associated risk factors with stroke development, analyze the effect of cigarette and water-pipe smoking, assess the prevalence of stroke survivors, develop a diagnosis score, assess care and outcomes, examine the utilization of thrombolytic therapy, and assess the post discharge medication prescription. ${ }^{15-21}$ However, it is unknown whether prescription patterns of antihypertensive medications are consistent with the guidelines regarding the effective management of high BP and we hypothesized that patients with AIS are often prescribed antihypertensive medications even though the BP readings do not meet the criteria for HTN treatment since in Lebanon there is absence of local guidelines for stroke management. The aim of this study is to describe and assess the frequency and the factors affecting overtreatment of HTN in AIS and evaluate the utilization of the different antihypertensive drugs in the emergency department (ED) in AIS.

\section{Methods}

\subsection{Study design \& study population}

This was a retrospective observational study conducted in different Lebanese hospitals (Rafik Hariri University Hospital, and Ain W Zein Medical Village) between February and May 2019. The medical records of 146 consecutive patients with AIS were reviewed for possible inclusion. The Institutional Review Board of the hospitals and the research committee at the faculty of pharmacy of the Lebanese International University approved the study protocol and informed consent from the patients was not required as the study was retrospective and did not cause any harm.

\subsection{Inclusion and exclusion criteria}

All adult patients admitted to the ED for acute ischemic stroke confirmed by a brain computed tomography-scan and followed by acute in-hospital treatment were included. Excluded from the analysis were patients whose brain scans haven't confirmed the diagnosis of ischemic stroke, received recombinant tissue-plasminogen activator, or had missing BP data in the medical charts.

\subsection{Data collection}

Medical records review was performed on site by the principal investigator, without interference or bias, using a structured data collection sheet. A structured questionnaire was used to gather data; and a pre-tested questionnaire was designed for this purpose. The information collected from the charts included demographic characteristics of patients, risk factors for stroke, systolic and diastolic BP at admission, 2 and $24 \mathrm{~h}$ post the intake of antihypertensive medications, and data about antihypertensive medications taken at home and at the ED. Patients were categorized into two groups: the first group was defined if patients had $\mathrm{BP}$ readings that were above the target treatment threshold and required further treatment and the second one was identified as those who had elevated BP readings that did not meet the target treatment criteria but were prescribed an antihypertensive medications. The latter group was termed as overtreatment group. Overtreatment was defined if the systolic blood pressure (SBP) and/or diastolic blood pressure (DBP) level upon admission was below $220 \mathrm{mmHg}$ and $120 \mathrm{mmHg}$ respectively and an antihypertensive medication was prescribed. According to AHA/ ASA guidelines recommendations, antihypertensive medication should be prescribed if the BP value is above $220 / 120 \mathrm{~mm} \mathrm{Hg}$ associated with end organ damage, and if the patient is candidate for thrombolysis with BP readings above $185 / 110 \mathrm{mmHg} .{ }^{8}$

Moreover, functional outcome at admission and discharge were assessed using the modified Rankin scale (mRS). Scores on the mRS range from 0 to 6 , with a score of 0 indicating no symptoms, a score of 5 indicating severe disability, and a score of 6 indicating death. A score of $0-2$ was considered of favorable stroke outcome and scores of 3 or more considered as poor stroke outcome. ${ }^{22}$

\subsection{Outcomes}

The primary outcome of this study was to describe the frequency of BP overtreatment in AIS. Secondary outcomes were to assess and evaluate factors that influence BP overtreatment including past medical history, medication history, patients' outcome and BP levels at baseline.

\subsection{Sample size calculation}

The Epi info software was used to calculate the minimal sample size required, considering a confidence interval of $95 \%$, prevalence of stroke in Lebanese population of $0.5 \%$, and $56 \%$ hypertension overtreatment according to ASA guidelines in Minnesota. ${ }^{23,24}$ The minimal sample size calculated was 93 adults and children; this figure was increased to allow for adequate bivariate and multivariable analyses, and also to take into account for missing data.

\subsection{Statistical analysis}

Statistical analysis was performed using the Statistical Package for the Social Science software (version 21). Descriptive statistics were used to describe patient characteristics and mean ( \pm standard deviation) for continuous variables. Comparison of proportions was performed using Pearson's Chi squared test and Fisher's exact test. In addition, student's t-test was performed to compare mean BP changes. Non-parametric tests replaced the parametric tests in case of non-homogeneity. The statistical analysis was adjusted for the missing values by accounting for the valid percentage in the results output. Multiple logistic regressions models were used to assess the association between past medical history and the $\mathrm{BP}$ overtreatment (taken as the dependent variable) that showed a pvalue $<0.2$ in the bivariate analysis. Potential confounders may be eliminated only if $\mathrm{p}$-value $>0.2$, in order to protect against residual confounding. All reported p-values were two-sided and values $<0.05$ were considered statistically significant.

\section{Results}

\subsection{Sociodemographic characteristics}

A total of 141 patients were enrolled in this study with a mean age \pm standard deviation of $76.89 \pm 11.258$ years and $43.3 \%$ were males. Most of the participants had normal body mass index $(37.1 \%)$, were married (85.9\%), did not smoke (74.6\%), did not consume water-wipe $(90.8 \%)$, and were alcohol free (94.8\%). Patients had different comorbidities with the most common disease being HTN (77.3\%), followed by stroke, tachycardia and hyperlipidemia (38.3\% each). Anti-hypertensive agents were the most previous concomitant drugs prescribed to the patients (66.4\%), followed by aspirin (42.6\%) (Table 1$)$.

\subsection{BP management according to AHA/ASA guidelines}

From the total enrolled patients, only $7.1 \%$ who met the criteria for 
Table 1

Patients' characteristics.

\begin{tabular}{|c|c|}
\hline Characteristics $(\mathrm{n}=141)$ & $\mathrm{N}(\%)$ \\
\hline Age (mean \pm standard deviation) & $76.89 \pm 11.528$ \\
\hline \multicolumn{2}{|l|}{ Gender } \\
\hline Male & $61(43.3 \%)$ \\
\hline Female & $80(56.7 \%)$ \\
\hline \multicolumn{2}{|l|}{ Body Mass Index } \\
\hline Underweight & $2(5.7 \%)$ \\
\hline Normal & $13(37.1 \%)$ \\
\hline Overweight & $9(25.7 \%)$ \\
\hline Obese & $11(31.4 \%)$ \\
\hline \multicolumn{2}{|l|}{ Marital Status } \\
\hline Married & $55(85.9 \%)$ \\
\hline Single & $3(4.7 \%)$ \\
\hline Divorced/widowed & $6(9.4 \%)$ \\
\hline \multicolumn{2}{|l|}{ Smokers } \\
\hline No & $100(74.6 \%)$ \\
\hline Yes & $34(25.4 \%)$ \\
\hline \multicolumn{2}{|l|}{ Water pipe } \\
\hline No & $108(90.8 \%)$ \\
\hline Yes & $11(9.2 \%)$ \\
\hline \multicolumn{2}{|l|}{ Alcohol } \\
\hline No & $128(94.8 \%)$ \\
\hline Yes & $7(5.2 \%)$ \\
\hline \multicolumn{2}{|l|}{ In hospital death } \\
\hline No & $136(96.5 \%)$ \\
\hline Yes & $5(3.5 \%)$ \\
\hline \multicolumn{2}{|l|}{ Past medical History } \\
\hline \multicolumn{2}{|l|}{ Hypertension } \\
\hline No & $32(22.7 \%)$ \\
\hline Yes & $109(77.3 \%)$ \\
\hline \multicolumn{2}{|l|}{ Stroke } \\
\hline No & $87(61.7 \%)$ \\
\hline Yes & $54(38.8 \%)$ \\
\hline \multicolumn{2}{|l|}{ Tachycardia } \\
\hline No & $87(61.7 \%)$ \\
\hline Yes & $54(38.3 \%)$ \\
\hline \multicolumn{2}{|l|}{ Hyperlipidemia } \\
\hline No & $87(61.7 \%)$ \\
\hline Yes & $54(38.3 \%)$ \\
\hline \multicolumn{2}{|l|}{ Diabetes Mellitus } \\
\hline No & $94(66.7 \%)$ \\
\hline Yes & $47(33.3 \%)$ \\
\hline \multicolumn{2}{|l|}{ Past Medication History } \\
\hline \multicolumn{2}{|l|}{ Vitamin $\mathrm{K}$ antagonist } \\
\hline No & $117(83 \%)$ \\
\hline Yes & $24(17 \%)$ \\
\hline \multicolumn{2}{|l|}{ Aspirin } \\
\hline No & $81(57.4 \%)$ \\
\hline Yes & $60(42.6 \%)$ \\
\hline \multicolumn{2}{|l|}{ Lipid lowering agents } \\
\hline No & $96(68.1 \%)$ \\
\hline Yes & $45(31.9 \%)$ \\
\hline \multicolumn{2}{|l|}{ Anti-hypertensive drugs } \\
\hline None & $47(33.6 \%)$ \\
\hline One and more & $93(66.4 \%)$ \\
\hline \multicolumn{2}{|l|}{ Overtreatment } \\
\hline No & $49(37.4 \%)$ \\
\hline Yes & $82(62.6 \%)$ \\
\hline
\end{tabular}

hypertension treatment guidelines recommendation, was not prescribed antihypertensive medication. On the other hand, out of 131 patients (92.9\%) who were not eligible for BP treatment according to AHA/ASA guidelines, 82 patients $(62.6 \%)$ were prescribed an antihypertensive medication, and thus were considered overtreated.

\subsection{Association between past medical, medication history, and patients' outcome with overtreatment}

The results showed that patients with HTN, hyperlipidemia and physically in-active were significantly overtreated with a p-value $<$ 0.05 . As for the relation between past medication history and overtreatment, patients on previous intake of anti-hypertensive agents were significantly overtreated with a p-value of 0.001 . As for the patient outcome, the results showed that patients with high mRS scores $(>2)$ at baseline were significantly overtreated with antihypertensive medications compared to $\mathrm{mRS}$ scores $<2$ (Table 2 ).

\subsection{Multivariate analysis}

Multivariate analysis was done to evaluate the relation of various independent variables with the BP overtreatment as dependent variable. The independent variables included were hypertension, hyperlipidemia, physically not active, anti-hypertensive agents, mRS at baseline, SBP and DBP at baseline, difference of SBP between baseline and after $2 \mathrm{~h}$, and difference of DBP between baseline and $24 \mathrm{~h}$. Results of multivariate analysis showed that the highest risk of overtreatment was shown significantly in patients who had HTN and who had high SBP at baseline with an OR of 4.589 and p-value of 0.017 and OR of 1.061 and p-value of 0.001 respectively as illustrated in Table 3 .

\section{Discussion}

This study was the first to describe and assess the frequency and the factors affecting overtreatment of BP in AIS. In our study involving patients with AIS, acute BP management was overall not consistent with recommendations by AHA/ASA guidelines. Around two-thirds of the patients with AIS present with elevated BP levels that are not above the target treatment threshold to warrant antihypertensive medication administration. Thus, aggressive treatment of HTN in AIS in this study may be due to approaching stroke as a hypertensive emergency associated with definitive organ damage which is managed by strict lowering of BP.

\subsection{Rates of overtreatment}

Our study revealed that few patients (7.1\%) presented with BP readings that meet the treatment recommendations according to ASA guidelines. Our results were consistent with Grise et al. where $6.3 \%$ met criteria for treatment. ${ }^{25}$ However, other studies reported higher numbers $(22 \%)$ of patients eligible for BP treatment with Lindenauer et al. ${ }^{26}$ Almost all patients who were eligible for BP treatment received an antihypertensive, and thus were consistent with treatment guidelines. Our results highlighted the fact that almost all patients ( 9 out of 10) met treatment recommendations and received antihypertensive

Table 2

Association between past medical history and overtreatment.

\begin{tabular}{lll}
\hline Diseases $(\mathrm{n}=82)$ & Overtreatment & P value \\
\hline Hypertension & $7(9 \%)$ & $<\mathbf{0 . 0 0 1}$ \\
No & $71(91 \%)$ & \\
Yes & $33(44.6 \%)$ & \\
Hyperlipidemia & $41(55.4 \%)$ & \\
No & & \\
Yes & $27(32.9 \%)$ & \\
Physically in-active & $55(67.1 \%)$ & $\mathbf{0 . 0 1}$ \\
No & $18(22 \%)$ & \\
Yes & $64(78 \%)$ & \\
Anti-hypertensive agents & & \\
No & $3(4.6 \%)$ & \\
Yes & $62(95.4 \%)$ & \\
Modified Rankin Scale at baseline & \\
$0-2$ & $32(51.6 \%)$ & \\
$>2$ & $30(48.4 \%)$ & \\
Modified Rankin Scale at discharge & & \\
$0-2$ & & \\
$>2$ & $74(93.7 \%)$ & \\
In hospital death & $5(6.3 \%)$ & \\
No & & \\
Yes & & \\
\hline
\end{tabular}

Pearson Chi squared test used and Fisher's exact test utilized if the cell count is less than 5 . 
Table 3

Multivariable logistic regression: taking the BP overtreatment as the dependent variable and all other factors as the independent variables.

\begin{tabular}{llll}
\hline Variables & $\begin{array}{l}\text { Odds } \\
\text { Ratio }\end{array}$ & $\begin{array}{l}95 \% \text { Confidence } \\
\text { interval }\end{array}$ & $\begin{array}{l}\text { P } \\
\text { value }\end{array}$ \\
\hline $\begin{array}{l}\text { Previous history of hypertension (Yes vs } \\
\text { No) }\end{array}$ & 4.589 & $1.313-16.038$ & $\mathbf{0 . 0 1 7}$ \\
$\begin{array}{l}\text { Modified Rankin Scale at baseline ( }>2 \text { vs } \\
0-2)\end{array}$ & 5.125 & $0.699-37.583$ & 0.108 \\
$\begin{array}{l}\text { Difference of systolic blood pressure } \\
\text { between baseline and after 2 h (High } \\
\text { vs Low) }\end{array}$ & 0.96 & $0.92-1.002$ & 0.064 \\
$\begin{array}{l}\text { Systolic blood pressure at baseline (High } \\
\text { vs Low) }\end{array}$ & 1.061 & $1.025-1.098$ & $\mathbf{0 . 0 0 1}$ \\
\hline
\end{tabular}

P-value $<0.05$ considered statistically significant. 31\% (Nagelkerke R-square) of the variance of the hypertension overtreatment can be explained by previous history of hypertension, Modified Rankin Scale at baseline, and systolic blood pressure at baseline and after treatment by $2 \mathrm{~h}$.

medication which is consistent with a study done in Cameroon that showed a high percentage of treatment adherence. ${ }^{27}$ On the other hand, other studies had reported higher rates of treatment in adherence ranging from $16 \%$ to $36.7 \%{ }^{28},{ }^{26},{ }^{25}$ Our results showed that the guidelines were broadly followed for patients who required BP treatment which is probably due to the fact that most physicians are aware of the detrimental effects of high BP in AIS. Nevertheless, in our study the guidelines were generally not applied to patients presenting with SBP $<220$ and/or DBP $<120 \mathrm{mmHg}$, since antihypertensive medications were often administered despite not meeting the BP thresholds.

BP overtreatment rate was observed to be $62.6 \%$ in this study. However, the latter study differs from our study since they studied only the as-needed medication use. Also, Grise et al. reported $68.5 \%$ overtreatment rate in the ED. ${ }^{27}$ This shows that overtreatment is a universal problem and not only confined to Lebanon. There are several possibilities to explain this discrepancy observed in the "real world" compared to the guidelines. First, management of BP in AIS has been a long debated topic with several studies showing divergent results. The results of recent randomized controlled trials designed to address whether treating BP in the acute setting has an effect on clinical outcomes which revealed that lowering BP can be beneficial, neutral or harmful. Therefore, guideline recommendations are not based on strong evidence leaving room for variances in prescription patterns. Second, physicians approach SBP levels above $180 \mathrm{mmHg}$ as hypertensive crisis and thus treating patients rapidly. Third, there is lack of data on whether home antihypertensive medications should be continued acutely. Fourth, since HTN is the most important risk factor of stroke, physicians believe that early treatment prevents recurrence and minimizes complications. Finally, the fear of end organ damage due to high BP might prompt physicians to administer an antihypertensive.

\subsection{Factors associated with overtreatment}

Our results revealed that overtreatment was significantly more common in patients with a previous history of hypertension and were at least on one antihypertensive drug before admission. This could be attributed to the lack of clarity regarding whether home antihypertensive medications in the acute setting should be withheld or continued. In addition, those who had mRS $>2$ upon admission were also significantly more likely to be overtreated. Indeed, patients with higher National Institutes of Health Stroke Scale scores were significantly more likely to be treated with antihypertensive medication. ${ }^{27}$

\subsection{Limitations}

First, the retrospective design of this study is associated with some missing data from patient's medical records and restricted in clearly evaluating the factors behind physicians choosing the antihypertensive medications. Second, no randomization process was done thus, leaving room for selection bias. Finally, our questionnaire did not collect data on whether the antihypertensive given was on as needed basis or scheduled basis and whether the home antihypertensive drugs were withheld or continued.

\section{Conclusion}

Overall, the present study reveals that ED management of BP was not consistent with the guidelines. Moreover, overtreatment is more evident in patients who are previously hypertensive and are on antihypertensive therapies. The results showed that physicians tend to overprescribe antihypertensive medications during acute ischemic stroke which raises the need to increase awareness about the appropriate implementation of the guidelines in Lebanese hospitals. These findings support the need for more research to fill identified gaps in evidence, improve treatment guidelines, and reduce variability in patient management. In the meantime, greater efforts should be made to educate physicians about the guidelines and areas of adherence.

\section{Ethical considerations}

The Institutional Review Board of each hospital site and the research committee of the school of pharmacy at the Lebanese International University approved the research project. The informed consent was waived as the study was conducted retrospectively and did not involve direct patient contact. The study respected patient's privacy and confidentiality; the collected data was anonymous and stored in a sealed envelope out of reach. The data and the questionnaire are provided for scientific reason upon request from the corresponding author.

\section{Declaration of competing interest}

The authors declare that they have no competing interests.

\section{Acknowledgements}

Funding: This research did not receive any specific grant from funding agencies in the public, commercial, or not-for-profit sectors.

\section{References}

1 Wajngarten M, Silva GS. Hypertension and stroke: update on treatment. Eur Cardiol. 2019 Jul 11;14(2):111-115.

2 Thom T, Haase N, Rosamond W, et al. Heart disease and stroke statistics-2006 update: a report from the American heart association statistics committee and stroke statistics subcommittee. Circulation. 2006 Feb 14;113(6):e85-151.

3 Gaciong Z, Siński M, Lewandowski J. Blood pressure control and primary prevention of stroke: summary of the recent clinical trial data and meta-analyses. Curr Hypertens Rep. 2013 Dec;15(6):559-574.

4 Gąsecki D, Coca A, Cunha P, et al. Blood pressure in acute ischemic stroke: challenges in trial interpretation and clinical management position of the ESH Working Group on Hypertension and the Brain. J Hypertens. 2018 Jun;36(6):1212-1221.

5 Lobanova I, Qureshi AI. Blood pressure goals in acute stroke-how low do you go? Curr Hypertens Rep. 2018 Apr 10;20(4):28.

6 José Castillo, Rogelio Leira, García María M, Joaquín Serena, Blanco Miguel, Antoni Dávalos. Blood pressure decrease during the acute phase of ischemic stroke is associated with brain injury and poor stroke outcome. Stroke. 2004 Feb 1;35(2): $520-526$.

7 Vemmos KN, Tsivgoulis G, Spengos K, et al. U-shaped relationship between mortality and admission blood pressure in patients with acute stroke. J Intern Med. 2004;255 (2):257-265.

8 Powers. Guidelines for the Early Management of Patients with Acute Ischemic Stroke: A Guideline for Healthcare Professionals from the. American Heart Association/American Stroke Association; 2018, 2018;54.

9 Aslanyan S, Fazekas F, Weir CJ, Horner S, Lees KR, GAIN International Steering Committee and Investigators. Effect of blood pressure during the acute period of ischemic stroke on stroke outcome: a tertiary analysis of the GAIN International Trial. Stroke. 2003 Oct;34(10):2420-2425.

10 Sare Gillian M, Ali Myzoon, Ashfaq Shuaib, Bath Philip MW. Relationship between hyperacute blood pressure and outcome after ischemic stroke. Stroke. 2009 Jun 1;40 (6):2098-2103. 
11 Stead LG, Gilmore RM, Decker WW, Weaver AL, Brown RD. Initial emergency department blood pressure as predictor of survival after acute ischemic stroke. Neurology. 2005 Oct 25;65(8):1179-1183.

12 Jauch EC, Saver JL, Adams HP, et al. Guidelines for the early management of patients with acute ischemic stroke: a guideline for healthcare professionals from the American Heart Association/American Stroke Association. Stroke. 2013 Mar;44(3): 870-947.

13 Shinohara Y, Yamaguchi T. Outline of the Japanese guidelines for the management of stroke 2004 and subsequent revision. Int J Stroke. 2008 Feb 1;3(1):55-62.

14 Zonneveld TP, Richard E, Vergouwen MD, et al. Blood pressure-lowering treatment for preventing recurrent stroke, major vascular events, and dementia in patients with a history of stroke or transient ischaemic attack. Cochrane Database Syst Rev. 2018 Jul 19;7, CD007858.

15 El-Hajj M, Salameh P, Rachidi S, Al-Hajje A, Lahoud N, Hosseini H. Stroke risk factors: a hospital-based case-control study in Lebanon. J R Soc Med. 2017;8(6): $1-10$.

16 El-Hajj M, Salameh P, Rachidi S, Al-Hajje A, Hosseini H. Cigarette and waterpipe smoking are associated with the risk of stroke in Lebanon. Clin Epidemiol Glob Health. 2019 Mar;9(1):62-70.

17 Lahoud N, Salameh P, Saleh N, Hosseini H. Prevalence of Lebanese stroke survivors: a comparative pilot study. Clin Epidemiol Glob Health. 2016 Sep 1;6(3):169-176.

18 El-Hajj M, Salameh P, Rachidi S, Al-Hajje A, Hosseini H. Development of a diagnosis score for stroke in the Lebanese population. Clin Epidemiol Glob Health. 2019 Mar 1;7 (1):79-87.
19 Lahoud N, Salameh P, Hosseini H, et al. Care and discharge outcome of acute stroke in Lebanon: a hospital-based study. J Nerv Ment Dis. 2018 Aug;206(8):637-643.

20 El Sayed MJ, El Zahran T, Tamim H. Acute stroke care and thrombolytic therapy use in a tertiary care center in Lebanon. Emerg Med Int. 2014;2014:1-6.

21 Malaeb D, Cherri S, Hallit S, Saade S, Hosseini H, Salameh P. Assessment of post discharge medication prescription among Lebanese patients with cerebral infarction: results of a cross-sectional study. Clin Neurol Neurosurg. 2020 Apr 1;191, 105674.

22 Broderick JP, Adeoye O, Elm J. Evolution of the modified Rankin scale and its use in future stroke trials. Stroke. 2017:48(7):2007-2012.

23 Lahoud N, Salameh P, Saleh N, Hosseini H. Prevalence of Lebanese stroke survivors: a comparative pilot study. J Epidemiol Glob Health. 2016;6(3):169-176.

24 Lakshminarayan K, Anderson DC, Borbas C, Duval S, Luepker RV. Blood pressure management in acute ischemic stroke. J Clin Hypertens. 2007 Jun;9(6):444-453.

25 Grise EM, Adeoye O, Lindsell C, et al. Emergency department adherence to AHA guidelines for blood pressure management in acute ischemic stroke. Stroke. 2012 Feb;43(2):557-559.

26 Lindenauer PK, Mathew MC, Ntuli TS, Pekow PS, Fitzgerald J, Benjamin EM. Use of antihypertensive agents in the management of patients with acute ischemic stroke. Neurology. 2004 Jul 27;63(2):318.

27 Kuate-Tegueu C, Dongmo-Tajeuna JJ, Doumbe J, Mapoure-Njankouo Y, Noubissi G, Djientcheu VDP. Management of blood pressure in acute stroke: comparison of current prescribing patterns with AHA/ASA guidelines in a Sub-Saharan African referral hospital. J Neurol Sci. 2017 Nov;382:137-141.

28 Lakshminarayan K, Anderson DC, Borbas C, Duval S, Luepker RV. Blood pressure management in acute ischemic stroke. J Clin Hypertens. 2007 Jun;9(6):444-453. 